\title{
A Symmetry-Based Method for the Determination of Vertebral Rotation in 3D
}

\author{
Tomaž Vrtovec, Franjo Pernuš, and Boštjan Likar \\ University of Ljubljana, Faculty of Electrical Engineering, Slovenia \\ \{tomaz.vrtovec, franjo.pernus, bostjan.likar\}@fe.uni-lj.si
}

\begin{abstract}
In the past, a number of methods were proposed for quantitative assessment of vertebral rotation from three-dimensional (3D) images. However, these methods were based on manual identification of distinctive anatomical landmarks, required manual determination of cross-sections from 3D images, and measured only axial vertebral rotation instead of the rotation in 3D. In this paper, we propose an automated method for quantitative assessment of vertebral rotation in 3D that is based on finding the planes of vertebral symmetry by matching image intensity gradients on both sides of each plane. The method was evaluated on 28 images of normal and pathological vertebrae, obtained by computed tomography (CT) and magnetic resonance (MR). For each vertebra, final angle displacements of 200 initial angle displacements, uniformly distributed within $30^{\circ}$ from manually obtained reference angles, were obtained. The results show that by the proposed method, vertebral rotation can be successfully estimated in $3 \mathrm{D}$ with an average accuracy of $1.0^{\circ}$ and precision of $0.5^{\circ}$.
\end{abstract}

\section{Introduction}

Vertebral rotation is important for the understanding of normal and pathological spine conditions. In the past, many methods for quantitative assessment of vertebral rotation were developed for two-dimensional (2D) X-ray images, while the measurements in three dimensions (3D) became possible with the development of 3D imaging techniques. Earlier methods were based on identifying a number of distinctive anatomical landmarks on vertebrae in both computed tomography (CT) and magnetic resonance (MR) axial cross-sections [1/234]. Recently, methods based on image analysis techniques have been proposed for measuring vertebral rotation in 3D. Rogers et al. proposed a method that measured the axial vertebral rotation between two axial MR [5] or CT [6] cross-sections by maximizing the correlation of intensities inside manually defined circular areas. Oblique CT cross-sections were used by Adam and Askin [7], who determined the axial vertebral rotation with the line that bisected the manually defined area around the vertebral body, so that the correlation of thresholded intensities in the bisected regions was maximal. Kouwenhoven et al. measured vertebral rotation in manually selected axial cross-sections from CT [8] and MR [9] images of normal spines. The rotation was defined with the line passing through the center

D. Metaxas et al. (Eds.): MICCAI 2008, Part I, LNCS 5241, pp. $9 4 2 \longdiv { - 9 5 0 } 2 0 0 8 .$

(C) Springer-Verlag Berlin Heidelberg 2008 
of the vertebral canal and the center of the anterior half of the vertebral body, both obtained by an automatic region growing segmentation technique. These methods required manual initialization of parameters, which may lead to errors because:

1. Manual identification of distinctive anatomical landmarks is difficult due to variable vertebral anatomical structure (e.g. pathological cases) and variable image quality (e.g. low image resolution).

2. Manual determination of oblique (reformatted) cross-sections consists of determining one or two rotation angles and requires a relatively difficult navigation through $3 \mathrm{D}$ images.

3. The vertebrae are in general rotated in $3 \mathrm{D}$, i.e. in sagittal, coronal and axial planes. In case of significant sagittal and/or coronal rotation, errors are induced in the measurement of axial rotation in $2 \mathrm{D}$ cross-sections.

To avoid these problems, we propose an automated method for quantitative assessment of vertebral rotation in 3D. The method is based on finding the planes of vertebral symmetry by matching image intensity gradients on both sides of each plane. The method can be applied to images of normal and pathological vertebrae, acquired either by CT or MR.

\section{Method}

\subsection{Vertebral Rotation and Anatomical Correspondence in 3D}

The rotation of a vertebra in a $3 \mathrm{D}$ image can be represented by the angles of rotation of the coordinate system $V$ of the vertebra around the axes of the coordinate system $I$ of the $3 \mathrm{D}$ image:

$$
\boldsymbol{\omega}=\left(\omega_{x}, \omega_{y}, \omega_{z}\right)
$$

The axes of the image (global) coordinate system $I$ and vertebral (local) coordinate system $V$ can be represented by the Cartesian unit vectors $\left\{\boldsymbol{e}_{I x}, \boldsymbol{e}_{I y}, \boldsymbol{e}_{I z}\right\}$ and $\left\{\boldsymbol{e}_{V x}, \boldsymbol{e}_{V y}, \boldsymbol{e}_{V z}\right\}$, respectively. The angles $\omega_{x}, \omega_{y}$ and $\omega_{z}$ then represent the rotation of the vertebral coordinate system $V$ around vectors $\boldsymbol{e}_{I x}$ (pitch), $\boldsymbol{e}_{I y}$ (roll) and $\boldsymbol{e}_{I z}$ (yaw), respectively.

If the origin of $V$ is located at the vertebral body center and $V$ is rotationally aligned with the vertebra in $I$, anatomically corresponding (symmetrical) parts of the vertebra can be observed within volumes of interest (VOIs) along positive/negative directions of each axis $\boldsymbol{e}_{V j}, j=x, y, z$, of $V$ (Fig. 1 1 ). This means that the vertebra is symmetrical in left/right $\left( \pm_{V x}\right)$ directions, and that the vertebral body is symmetrical in anterior/posterior $\left( \pm \boldsymbol{e}_{V y}\right)$ and upward/downward $\left( \pm \boldsymbol{e}_{V z}\right)$ directions. Because the observed correspondences decrease with increasing rotation between $V$ and $I$, the angles $\boldsymbol{\omega}$ of vertebral rotation can be obtained by evaluating these anatomical correspondences of vertebral structures. It is important to note that the origin of $V$ is located at the vertebral body center and not at the vertebral anatomical center of rotation, which was reported to be in 


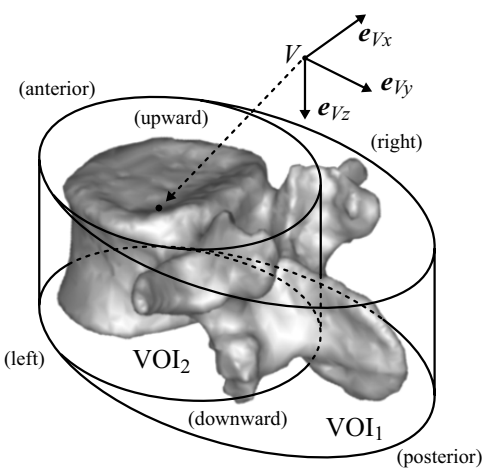

(a)

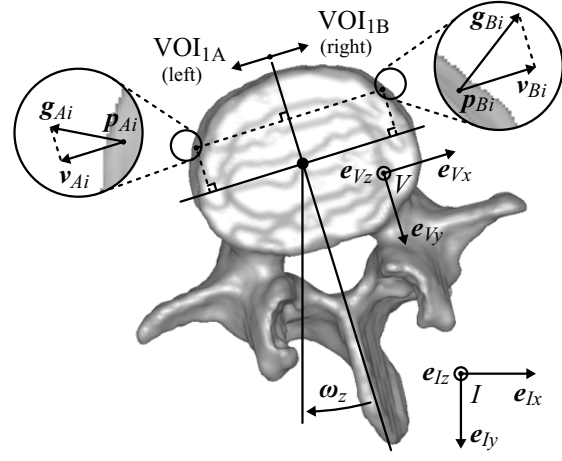

(b)

Fig. 1. (a) The vertebral coordinate system $V$ and the observed volumes of interest ( $\mathrm{VOI}_{1}$ contains the whole vertebra, $\mathrm{VOI}_{2}$ contains only the vertebral body). (b) Example of anatomical correspondence in left/right directions $\left(S_{x}\right)$, shown for a symmetrical pair of points $\boldsymbol{p}_{A i}$ and $\boldsymbol{p}_{B i}$ inside $\mathrm{VOI}_{1}$.

the mid-sagittal plane at the anterior wall of the vertebral canal 5] or at the center of the vertebral canal 8. However, the rotation angles do not depend on the location of the origin of $V$, but on the axes of $V$, i.e. on the vectors $\boldsymbol{e}_{V j}$, $j=x, y, z$, that have to be rotationally aligned with the vertebra in $I$.

\subsection{Determination of Vertebral Rotation in 3D}

The angles $\boldsymbol{\omega}$ of vertebral rotation can be determined by finding the planes of vertebral symmetry that define maximal anatomical correspondences. For each axis $\boldsymbol{e}_{V j}, j=x, y, z$, we propose to measure the correspondences of two halves of a $\mathrm{VOI}\left(\mathrm{VOI}_{A}\right.$ and $\mathrm{VOI}_{B}$, Fig. 10) by:

$$
S_{j}(\mathrm{VOI})=\frac{\sum_{i=1}^{N}\left|\boldsymbol{v}_{A i}\right| \cdot\left|\boldsymbol{v}_{B i}\right| \cdot f}{\sum_{i=1}^{N}\left|\boldsymbol{v}_{A i}\right| \cdot \sum_{i=1}^{N}\left|\boldsymbol{v}_{B i}\right|} ; \quad f=\left\{\begin{array}{ll}
1 ; & \boldsymbol{v}_{A i} \cdot \boldsymbol{v}_{B i}<0 \\
0 ; & \text { otherwise }
\end{array},\right.
$$

where $f$ is the weighting function, and $\boldsymbol{v}_{A i}=\operatorname{proj}_{\boldsymbol{e}_{V j}} \boldsymbol{g}_{A i}$ and $\boldsymbol{v}_{B i}=\operatorname{proj}_{\boldsymbol{e}_{V j}} \boldsymbol{g}_{B i}$ are the projections of the intensity gradient vectors $\boldsymbol{g}_{A i}=\operatorname{grad} I\left(\boldsymbol{p}_{A i}\right)$ and $\boldsymbol{g}_{B i}=$ $\operatorname{grad} I\left(\boldsymbol{p}_{B i}\right)$ in the coordinate system $I$ to the unit vector $\boldsymbol{e}_{V j}, j=x, y, z$, of the coordinate system $V$ at symmetrical pair of points $\boldsymbol{p}_{A i}$ and $\boldsymbol{p}_{B i}$, respectively. A total of $N$ pairs of symmetrical points exist inside each VOI. By projecting the gradient vectors to the axis $\boldsymbol{e}_{V j}, j=x, y, z$, and by applying the weighting function $f$, we retain the gradient components $\boldsymbol{v}_{A i}$ and $\boldsymbol{v}_{B i}$ that are relevant for defining the vertebral symmetry in the direction of the axis $\boldsymbol{e}_{V j}$.

It is expected that the anatomical structures in VOI halves will correspond maximally when $V$ is rotationally aligned with the vertebra in $I$. Therefore, the 

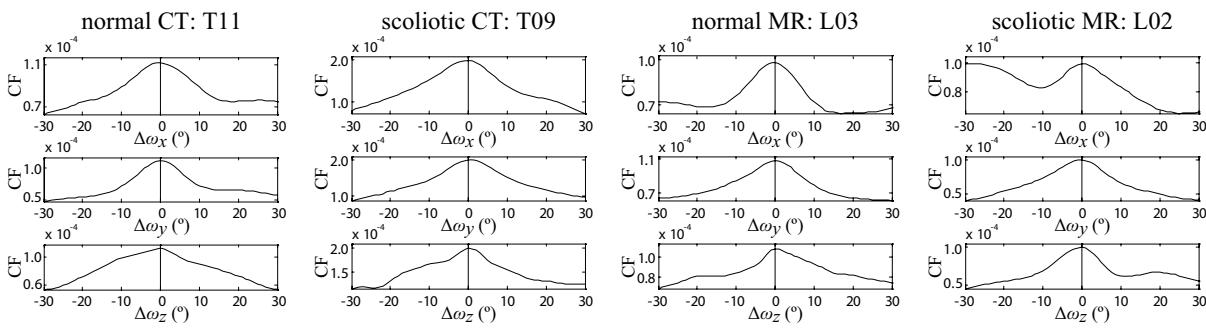

Fig. 2. Behavior of the criterion function $C F$ for different vertebrae, obtained by independently varying the angles $\boldsymbol{\omega}=\left(\omega_{x}, \omega_{y}, \omega_{z}\right)$ for $-30^{\circ} \div 30^{\circ}$ from the reference angles $\boldsymbol{\omega}_{R}$
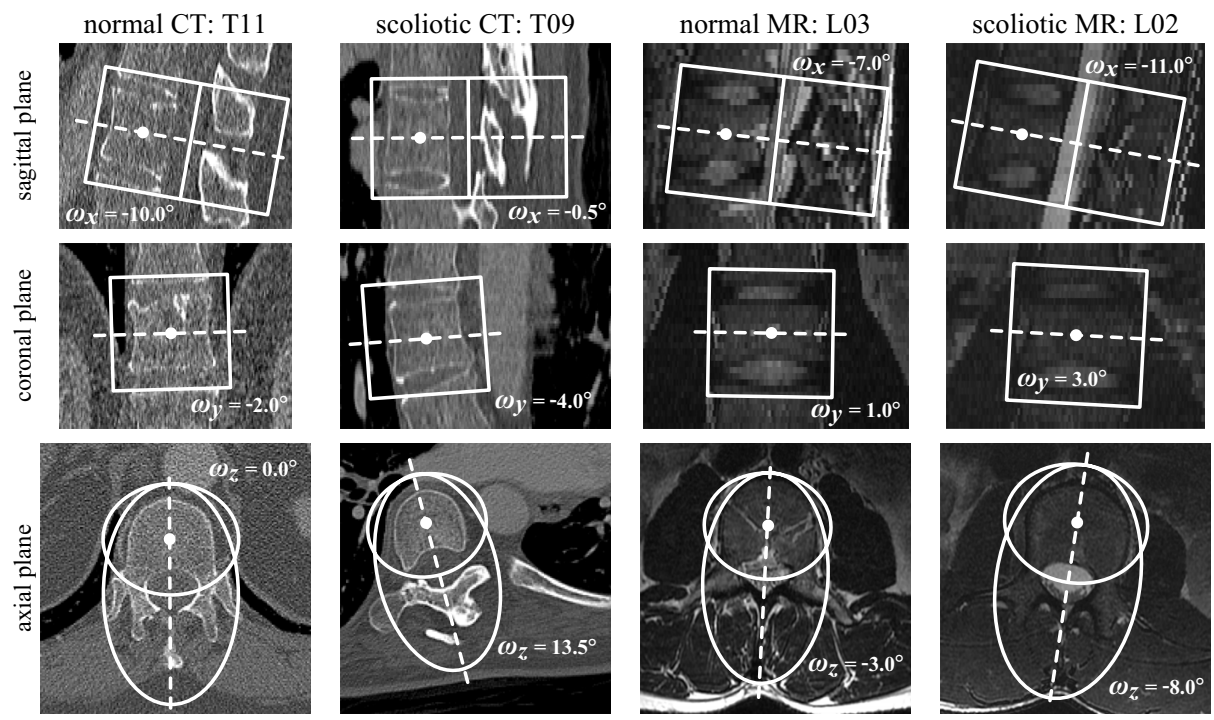

Fig. 3. Examples of the determination of reference angles $\boldsymbol{\omega}_{R}$ and the initialization of VOIs in $3 \mathrm{D}$, shown for different vertebrae

rotation of the coordinate system $V$, i.e. the angles $\boldsymbol{\omega}$ of vertebral rotation, can be found by maximizing the following criterion function:

$$
C F=S_{x}\left(\mathrm{VOI}_{1}\right)+S_{y}\left(\mathrm{VOI}_{2}\right)+S_{z}\left(\mathrm{VOI}_{2}\right) .
$$

Figure 2 illustrates the behavior of the criterion function $C F$, obtained by independently varying the angles $\omega_{x}, \omega_{y}$ and $\omega_{z}$ from reference angle values. The large capture ranges and distinctive maxima of $C F$ indicate that the proposed criterion function is feasible for the estimation of vertebral rotation angles. 


\section{$3 \quad$ Experiments and Results}

\subsection{Data and Experiments}

The anatomical structure of an arbitrary vertebra may significantly differ from the "normal" structure. This usually occurs in spinal diseases (e.g. scoliosis, hyper-kyphosis/lordosis) due to pathological vertebral growth. Besides, the rotation of an arbitrary vertebra in the 3D image depends on the orientation of image acquisition planes and on the position of the patient in scanner. To analyze the performance of the proposed method with respect to different vertebral shapes, we used 20 vertebrae from images of normal spines (CT: 10, voxel size $0.7 \times 0.7 \times 1.0 \mathrm{~mm}^{3} ; \mathrm{MR}: 10$, voxel size $\left.0.4 \times 0.4 \times 3.0 \mathrm{~mm}^{3}\right)$ and 8 vertebrae from images of scoliotic spines (CT: 4 , voxel size $0.6 \times 0.6 \times 1.0 \mathrm{~mm}^{3}$, strong right thoracolumbar curve; MR: 4 , voxel size $0.4 \times 0.4 \times 3.0 \mathrm{~mm}^{3}$, mild left lumbar curve). The scoliotic vertebrae were located around the apex of the deformity (usually most rotated and different from the normal shape). For each vertebra, reference rotation angles $\boldsymbol{\omega}_{R}$ were determined by manually defining the vertebral body center and by aligning the VOIs with the vertebra in the sagittal, coronal and axial cross-sections. The size of the VOIs were based on vertebral morphometric data, so that $\mathrm{VOI}_{1}$ and $\mathrm{VOI}_{2}$ contained the whole vertebra and the vertebral body, respectively (Figs. 1a, 3). Before computing the intensity gradient vectors $(\operatorname{grad} I)$ in $3 \mathrm{D}$, the images were blurred with a Gaussian filter $(\sigma=3 \mathrm{~mm})$. For each vertebra, the origin of the vertebral coordinate system $V$ was initialized in the manually defined vertebral body center. To study the influence of different vertebral orientations, we initialized the orientation of the vertebral coordinate system $V$ in angles that were for $\Delta \boldsymbol{\omega}$ displaced from the obtained reference angles $\boldsymbol{\omega}_{R}$, thus simulating an arbitrary initialization of the orientation of the vertebral coordinate system $V$. For each vertebra, 200 initial displacements were applied that were uniformly distributed within $|\Delta \boldsymbol{\omega}|=\left(\Delta \omega_{x}^{2}+\Delta \omega_{y}^{2}+\Delta \omega_{z}^{2}\right)^{1 / 2}=30^{\circ}$, resulting in a total of 5600 displacements for all vertebrae. For each initial angle displacement, the angles $\boldsymbol{\omega}$ were found by maximizing the criterion function (Eq. 3) with the Powell's optimization method.

\subsection{Results}

The results of the experiments are presented in Table 1. A single assessment of the rotation angles was considered successful if it resulted in an angle displacement less than $|\Delta \boldsymbol{\omega}|=2^{\circ}$ (i.e. $2.0^{\circ}$ for one or $1.1^{\circ}$ for each angle) from the reference angles $\boldsymbol{\omega}_{R}$. The results show that for most vertebrae, the success rate was equal or close to $100 \%$ if the initial angle displacement was below $10^{\circ}$ (i.e. $10^{\circ}$ for one or $5.8^{\circ}$ for each angle). For successful experiments, the average angle displacement was $\overline{|\Delta \boldsymbol{\omega}|}=0.95^{\circ}\left(\overline{\left|\Delta \boldsymbol{\omega}_{x}\right|}=0.58^{\circ}, \overline{\left|\Delta \boldsymbol{\omega}_{y}\right|}=0.53^{\circ}, \overline{\left|\Delta \boldsymbol{\omega}_{z}\right|}=0.53^{\circ}\right)$ and the average standard deviation was $\left|\sigma_{\Delta \omega}\right|=0.47^{\circ}\left(\sigma_{\Delta \omega_{x}}=0.28^{\circ}, \sigma_{\Delta \omega_{y}}=0.26^{\circ}\right.$, $\sigma_{\Delta \omega_{z}}=0.26^{\circ}$ ), which represent the accuracy and the precision of the method, respectively. 
Table 1. Median of 200 final angle displacements and success rates for three initial displacement ranges

\begin{tabular}{|c|c|c|c|c|c|c|c|c|}
\hline \multirow{2}{*}{\multicolumn{2}{|c|}{$\begin{array}{c}\text { Vertebra } \\
\text { image } \\
\text { (level) }\end{array}$}} & \multicolumn{4}{|c|}{$\begin{array}{c}\text { Median angle displacement } \\
\left({ }^{\circ}\right)\end{array}$} & \multicolumn{3}{|c|}{$\begin{array}{l}\text { Success rate } \\
\left(|\Delta \omega|<2^{\circ}\right)(\%)\end{array}$} \\
\hline & & $\Delta \omega_{x}$ & $\Delta \omega_{y}$ & $\Delta \omega_{z}$ & $|\Delta \boldsymbol{\omega}|$ & $0^{\circ} \div 10^{\circ}$ & $10^{\circ} \div 20^{\circ}$ & $20^{\circ} \div 30^{\circ}$ \\
\hline \multirow{10}{*}{ 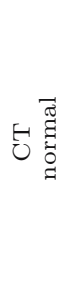 } & T07 & -1.0 & 1.1 & -0.9 & 1.7 & 70 & 64 & 46 \\
\hline & T08 & -0.7 & -0.2 & 0.1 & 0.8 & 100 & 100 & 98 \\
\hline & T09 & -0.3 & -0.3 & -1.3 & 1.5 & 94 & 93 & 80 \\
\hline & $\mathrm{T} 10$ & -0.5 & -0.1 & -1.3 & 1.5 & 86 & 73 & 71 \\
\hline & T11 & 0.3 & 0.1 & 0.0 & 0.4 & 100 & 100 & 93 \\
\hline & $\mathrm{T} 12$ & -0.2 & 0.2 & -0.4 & 0.5 & 100 & 100 & 90 \\
\hline & L01 & 0.5 & -1.5 & -0.6 & 1.8 & 86 & 88 & 61 \\
\hline & L02 & 0.9 & -0.6 & -0.5 & 1.2 & 100 & 97 & 72 \\
\hline & L03 & 1.2 & 0.3 & 0.5 & 1.3 & 98 & 97 & 67 \\
\hline & L04 & 0.0 & 0.0 & 0.2 & 0.4 & 86 & 85 & 55 \\
\hline \multirow{4}{*}{ 卢: } & T07 & -0.1 & -0.8 & -1.3 & 1.5 & 98 & 76 & 54 \\
\hline & T08 & -1.3 & -0.7 & -0.4 & 1.8 & 84 & 61 & 30 \\
\hline & T09 & -0.5 & 0.2 & 0.4 & 0.7 & 98 & 100 & 93 \\
\hline & T10 & 0.2 & 2.3 & 0.1 & 2.4 & 20 & 16 & 13 \\
\hline \multirow{10}{*}{$\underset{\Sigma}{2}$} & T07 & 0.0 & -0.3 & 0.5 & 0.6 & 89 & 94 & 54 \\
\hline & T08 & -1.8 & -0.4 & 0.4 & 2.2 & 52 & 36 & 28 \\
\hline & T09 & -1.2 & -0.2 & 0.5 & 1.4 & 100 & 81 & 55 \\
\hline & T10 & 0.5 & 0.9 & -0.3 & 1.2 & 100 & 94 & 80 \\
\hline & $\mathrm{T} 11$ & 0.2 & -0.2 & 0.0 & 0.3 & 98 & 100 & 67 \\
\hline & T12 & 1.5 & 0.6 & 0.2 & 1.6 & 100 & 100 & 77 \\
\hline & L01 & 1.0 & -0.2 & -0.2 & 1.1 & 98 & 97 & 71 \\
\hline & L02 & -0.5 & -0.8 & -0.2 & 1.0 & 100 & 100 & 83 \\
\hline & L03 & -0.1 & 0.1 & 0.1 & 0.3 & 100 & 99 & 57 \\
\hline & L04 & -0.2 & -0.1 & 0.5 & 0.6 & 100 & 82 & 65 \\
\hline \multirow{4}{*}{ 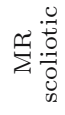 } & L01 & 0.2 & 0.5 & -0.1 & 1.0 & 94 & 60 & 29 \\
\hline & L02 & 0.1 & -0.6 & -0.5 & 0.8 & 100 & 73 & 52 \\
\hline & L03 & 0.1 & -0.4 & 1.3 & 1.5 & 100 & 82 & 36 \\
\hline & L04 & -0.2 & -0.7 & -0.1 & 1.2 & 98 & 93 & 59 \\
\hline
\end{tabular}

Figure 4 shows the scatter diagrams of the initial and final angle displacements for vertebrae that performed best and worst inside each group of normal CT, scoliotic CT, normal MR and scoliotic MR vertebrae (shown in bold in Tab. 1). It can be observed that the final displacements tend to converge to a value that is not equal to $0^{\circ}$, which means that the final angles were not equal to the reference angles. These constant displacements represent systematic errors, and when they were above $2^{\circ}$, the experiments were not considered successful. However, these systematic errors may originate in the reference angles $\boldsymbol{\omega}_{R}$, which were determined manually and may therefore not represent the "true" vertebral rotation. For example, although poor success rates are reported for the T10 scoliotic CT vertebra (Tab. 1), the scatter diagram (Fig. 4) shows that the angles always converged to approximately the same angle displacements with the median of $2.4^{\circ}$. This systematic error was mostly caused by the angle $\omega_{y}$, which resulted in approximately $-11^{\circ}$, while the reference angle was $-9^{\circ}\left(\Delta \omega_{y}=2.3^{\circ}\right)$. After examining this case in detail, we concluded that the reference angle $\omega_{y}$ could not be reliably defined manually due to the strong scoliotic nature of the vertebral anatomical structure, i.e. relatively strong shear of the vertebral body. 

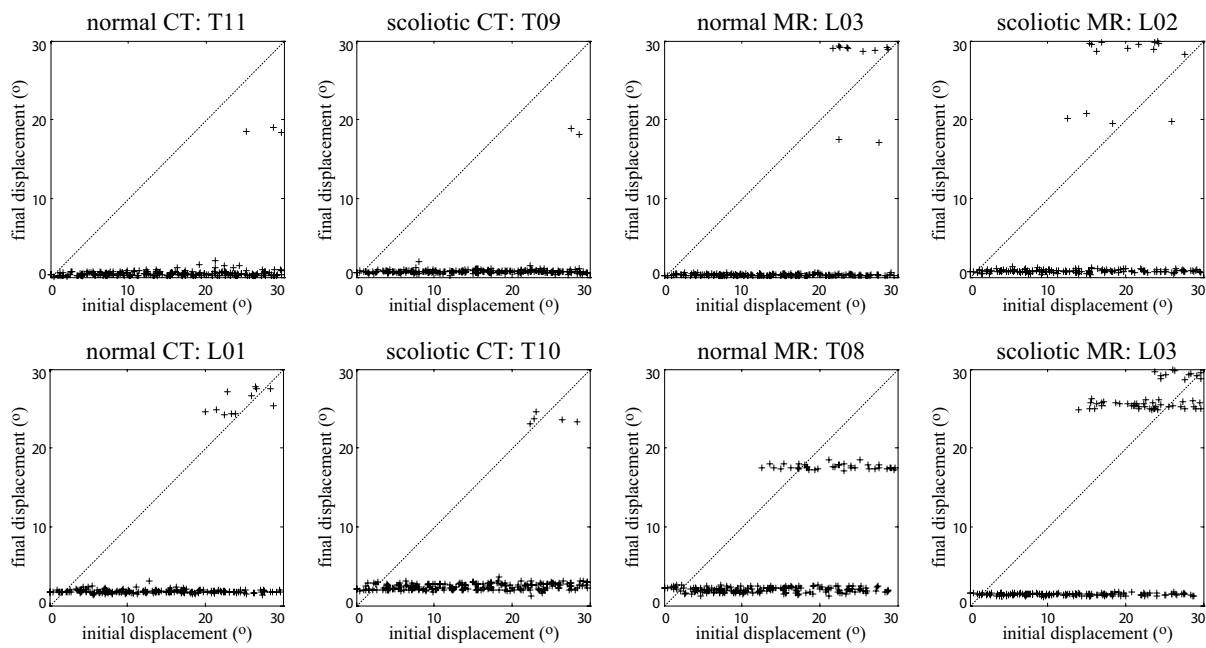

Fig. 4. Scatter diagrams of 200 initial and final angle displacements, shown for different vertebrae

\section{Discussion and Conclusion}

We proposed an automated method for quantitative assessment of vertebral rotation in $3 \mathrm{D}$. The relation between the image and vertebral coordinate systems was obtained by matching image intensity gradients that, when the vertebral coordinate system is aligned with the vertebra, define the symmetry of the vertebral anatomical structure. The method proved to be applicable to normal and pathological vertebrae, obtained from CT or MR images.

The results of axial vertebral rotation (i.e. angle $\omega_{z}$ ) are comparable to other methods that were based on image analysis techniques. Measurement errors of $0.2^{\circ}$ were reported for the method of Rogers et al. [56]. Adam and Askin [7] claimed their method to be relatively insensitive to image thresholding, however, they reported that different intensity thresholds caused a $2.8^{\circ}$ difference in rotation. Kouwenhoven et al. 89, reported the standard deviation of their method to be around $1^{\circ}$. By applying the proposed method, we achieved around $0.5^{\circ}$ accuracy and $0.3^{\circ}$ precision for the axial vertebral rotation. For the measurements of vertebral rotation in $3 \mathrm{D}$, the accuracy and precision of the proposed method were around $1.0^{\circ}$ and $0.5^{\circ}$, respectively.

The manual determination of reference rotation angles, required to assess the accuracy of any method, is usually based on the orientation of vertebral body and spinous process, shape of vertebral canal and other vertebral anatomical features. However, such features do not always represent a good anchor, as the anatomical structures are not always perfectly symmetrical and oriented in the same direction across the whole vertebra, resulting in different reference angles for different reference features. The reference angles have to be therefore 
considered with caution, as they may not represent the "true" rotation of the vertebra. The proposed method, on the other hand, estimates the vertebral rotation by finding the maximal possible symmetry of the entire vertebra. To conclude, the benefits of the method are at least the following:

1. The vertebral rotation can be defined with high accuracy and precision in $3 \mathrm{D}$, i.e. all three angles of rotation $\omega_{x}, \omega_{y}$ and $\omega_{z}$ around the three coordinate system axes are obtained. Errors due to projection of the vertebra onto a $2 \mathrm{D}$ plane are therefore eliminated.

2. The method may be used to determine the "true" ("gold standard") vertebral rotation, as the symmetry of the entire vertebral anatomical structure may represent an objective reference feature.

The symmetry of the vertebral anatomical structure may also be used to determine the reference center of rotation of the vertebral coordinate system. We are currently developing a method for robust determination of vertebral body centers in $\mathrm{CT}$ and MR images, which may allow an accurate determination of vertebral rotation and position in $3 \mathrm{D}$.

\section{Acknowledgements}

This work has been supported by the Ministry of Higher Education, Science and Technology, Slovenia, under grants P2-0232, L2-7381, L2-9758, and J2-0716. The authors would like to thank the Image Sciences Institute, Utrecht, The Netherlands, and the Commonwealth Scientific and Industrial Research Organisation (CSIRO), Australia, for providing the CT and MR images used in this study.

\section{References}

1. Aaro, S., Dahlborn, M.: Estimation of vertebral rotation and the spinal and rib cage deformity in scoliosis by computer-tomography. Spine 6(5), 460-467 (1981)

2. Ho, E., Upadhyay, S., Chan, F., Hsu, L., Leong, J.: New methods of measuring vertebral rotation from computed tomographic scans: An intraobserver and interobserver study on girls with scoliosis. Spine 18(9), 1173-1177 (1993)

3. Krismer, M., Sterzinger, W., Christian, H., Frischhut, B., Bauer, R.: Axial rotation measurement of scoliotic vertebrae by means of computed tomography scans. Spine 21(5), 576-581 (1996)

4. Göçen, S., Havitçioglu, H., Alici, E.: A new method to measure vertebral rotation from CT scans. European Spine Journal 8(4), 261-265 (1999)

5. Rogers, B., Haughton, V., Arfanakis, K., Meyerand, E.: Application of image registration to measurement of intervertebral rotation in the lumbar spine. Magnetic Resonance in Medicine 48(6), 1072-1075 (2002)

6. Rogers, B., Wiese, S., Blankenbaker, D., Meyerand, E., Haughton, V.: Accuracy of an automated method to measure rotations of vertebrae from computerized tomography data. Spine 30(6), 694-696 (2005) 
7. Adam, C., Askin, G.: Automatic measurement of vertebral rotation in idiopathic scoliosis. Spine 31(3), 80-83 (2006)

8. Kouwenhoven, J.W., Vincken, K., Bartels, L., Castelein, R.: Analysis of preexistent vertebral rotation in the normal spine. Spine 31(13), 1467-1472 (2006)

9. Kouwenhoven, J.W.M., Bartels, L., Vincken, K., Viergever, M., Verbout, A., Delhaas, T., Castelein, R.: The relation between organ anatomy and pre-existent vertebral rotation in the normal spine: Magnetic resonance imaging study in humans with situs inversus totalis. Spine 32(10), 1123-1128 (2007) 